\title{
Thyroglobulin Type-1 Domain Protease Inhibitors Exhibit Specific Expression in the Cortical Ooplasm of Vitellogenic Rainbow Trout Oocytes
}

\author{
ANTONY W. WOOD, ${ }^{1 *}$ JACQUELINE MATSUMOTO ${ }^{2}{ }^{2}$ AND GLEN VAN DER KRAAK ${ }^{2}$ \\ ${ }^{1}$ Department of Molecular, Cellular and Developmental Biology, University of Michigan, Ann Arbor, Michigan \\ ${ }^{2}$ Department of Zoology, University of Guelph, Guelph, Ontario, Canada
}

\begin{abstract}
The synthesis, uptake, and processing of yolk proteins remain poorly described aspects of oviparous reproductive development. In this study, we report the identification and characterization of two protease inhibitors in rainbow trout ovary whose expression and distribution are directly associated with yolk protein uptake in vitellogenic oocytes. The first transcript, termed "oocyte protease inhibitor-1" (OPI-1), is predicted to encode a $9.1 \mathrm{kDa}, 87$ amino acid protein containing a single thyroglobulin type-1 (TY) domain, identifying it as a putative TY domain inhibitor. The second transcript, termed $\mathrm{OPI}-2$, is predicted to encode an $18.3 \mathrm{kDa}, 173$ amino acid protein with two similar, but not identical, TY domains. Messenger RNA expression of both genes was first detected in ovarian tissues at the onset of vitellogenesis, and persisted throughout the vitellogenic growth phase. We did not detect expression of either gene in previtellogenic ovaries, nor in any somatic tissues examined. Expression of OPI-1 mRNA was significantly reduced in atretic follicles as compared to healthy vitellogenic follicles, suggesting a downregulation of inhibitor expression during oocyte atresia. Western immunoblot analyses of whole yolk from vitellogenic oocytes revealed the presence of two immunoreactive proteins that corresponded to the predicted sizes of OPI- 1 and $\mathrm{OPI}-2$. We detected strong crossreactivity of this antiserum with specific vesicles in the cortical ooplasm of vitellogenic oocytes, in regions directly associated with vitellogenin processing. The identification of OPI- 1 and OPI-2 provides new evidence for the expression of multiple TY domain protease inhibitors likely involved in the regulation of yolk processing during oocyte growth in salmonids. Mol. Reprod. Dev. 69: 205214, 2004. ๑ 2004 Wiley-Liss, Inc.
\end{abstract}

Key Words: vitellogenesis; follicle; oocyte; protease inhibitors; fish

\section{INTRODUCTION}

In oviparous vertebrates, oocyte growth is dependent upon the coordinated synthesis, uptake, processing, and storage of yolk proteins, derived primarily from the hepatic precursor vitellogenin. This suite of biochemical processes, collectively known as vitellogenesis, is essen- tial for the production of oocytes with sufficient yolk reserves to metabolically sustain the initial phases of embryonic and larval growth (Tata and Smith, 1979; Valle, 1993). Understanding the physiological and biochemical regulation of vitellogenesis thus remains a theme of considerable interest among reproductive biologists.

Much of what we currently know about vitellogenesis in vertebrates derives from studies in teleost fishes (Tyler and Sumpter, 1996). In teleosts, as in other vertebrates, vitellogenin is synthesized by the liver under estrogenic stimulation, secreted into the general circulation, and sequestered by developing oocytes via receptor-mediated endocytosis (Tyler et al., 1990, 1991). Subsequent intraoocytic proteolysis of vitellogenin generates an assortment of vesicular lipoproteins, (e.g., lipovitellins); these coalesce to form yolk platelets that are stored in the oocyte for the duration of oocyte development (Wallace and Selman, 1985; Carnevali et al., 1999a,b). After ovulation and fertilization, latent endogenous proteases are activated to initiate hydrolysis of the yolk platelets; the resultant release of lipids and free amino acids provides metabolic substrate for embryonic growth (Krieger and Fleig, 1999; Carnevali et al., 1999a; Hiramatsu et al., 2002).

There is considerable evidence to suggest that the proteolytic processing of both vitellogenin and yolk platelets is mediated by cathepsins, a diverse family of serine, aspartate, and cysteine proteases. Cathepsins have been implicated in yolk processing in fishes (Kestemont et al., 1999; Carnevali et al., 1999a,b; Kwon et al., 2001; Hiramatsu et al., 2002; Wood and Van Der Kraak, 2003), amphibians (Yoshizaki and Yonezawa, 1996), reptiles (De Stasio et al., 1999), birds (Gerhartz et al., 1999), and insects (Yamamoto et al.,

Grant sponsor: Natural Sciences and Engineering Research Council (NSERC) of Canada; Grant sponsor: Ontario Graduate Scholarship Program.

*Correspondence to: Antony W. Wood, Department of Molecular, Cellular and Developmental Biology, University of Michigan, Ann Arbor, MI 48109. E-mail: antonyw@umich.edu

Received 15 January 2004; Accepted 11 March 2004

Published online in Wiley InterScience (www.interscience.wiley.com). DOI $10.1002 / \mathrm{mrd} .20118$ 
1994; Ribolla et al., 2001), suggesting an evolutionarily conserved role for these enzymes in vitellogenin and yolk processing.

Despite evidence invoking cathepsins as modulators of yolk processing, there is little available information on the mechanisms by which these enzymes are regulated. Conventional protease inhibitors (e.g., cystatins) have been characterized in ovarian tissues (Coffman and Goetz, 1998; Li et al., 1998; Curry and Wheeler, 2002), but to our knowledge, none has been shown to be directly involved in regulating yolk proteolysis. However, the recent identification of "egg cysteine protease inhibitor" (ECI) in chum salmon provided new evidence for the existence of oocyte-specific protease inhibitors in fish (Yamashita and Konagaya, 1991). Chum salmon ECI (purified from ovulated eggs) exhibited inhibitory activity against cathepsins and cathepsin-like cysteine proteases in vitro, but was structurally unrelated to conventional cysteine protease inhibitors (Yamashita and Konagaya, 1991, 1996).

The defining feature of ECI is a cysteine-rich thyroglobulin type-1 (TY) domain, characteristic of a protein group recently termed "thyroglobulin type-1 domain protease inhibitors" (Molina et al., 1996; Lenarcic et al., 1997). A cDNA encoding a putatively homologous TY domain inhibitor (oocyte cysteine protease inhibitor, OCPI) was recently cloned from brook trout ovarian tissues (Bobe and Goetz, 2001), and shown to exhibit ovary-specific expression. These expression data, however, were derived almost exclusively from periovulatory ovarian tissues, thus there is no information on the expression of this gene during the prolonged vitellogenic phase of oocyte development, during which the specific regulation of oocyte proteases is required for proper oocyte development.

The ability of chum salmon ECI to inhibit cathepsins (Yamashita and Konagaya, 1991), and recent studies demonstrating regulated expression of cathepsins and other proteases in developing and degenerating teleost oocytes (Kestemont et al., 1999; Carnevali et al., 1999a; Kwon et al., 2001; Wood and Van Der Kraak, 2003), led us to hypothesize that ovarian TY domain inhibitors function to regulate yolk processing enzymes during vitellogenic oocyte development. We sought to test this hypothesis by cloning the homolog of ECI in rainbow trout, and characterizing its expression throughout vitellogenic oocyte development. We predicted that the expression of this putative TY domain inhibitor would temporally and spatially correlate with yolk protein deposition in vitellogenic oocytes. Furthermore, we predicted a downregulation of its expression in degenerating (atretic) oocytes, in which a detectable increase in cathepsin-like enzyme activities has recently been demonstrated (Kestemont et al., 1999; Wood and Van Der Kraak, 2003). These efforts led to the fortuitous identification of a second cDNA encoding a novel TY domain inhibitor that resembles of tandem duplication of the original target gene. Herein, we use RT-PCR and immunocytochemistry to describe the temporal and spatial expression patterns of these putatively para- logous protease inhibitors in the developing rainbow trout ovary.

\section{MATERIALS AND METHODS Commercial Reagents}

Oligonucleotide primers, SuperScript ${ }^{\mathrm{TM}}$ II reverse transcriptase, GeneRacer ${ }^{\mathrm{TM}}$ reagents, and RNase $\mathrm{H}$ were purchased from Invitrogen (Carlsbad, CA). Random hexamer primers were from Promega (Madison, WI). PCR nucleotide mix (dNTPs), dithiothreitol (DTT), first strand synthesis buffer (5X RT buffer), PCR reaction buffer $(10 \times$ PCR buffer), and TAq DNA polymerase (5 U/ $\mu \mathrm{l}$ ) were from Roche Molecular Biochemicals (Laval, PQ). RNase inhibitor (RNAguard ${ }^{\mathrm{TM}}, 25 \mathrm{U} / \mu \mathrm{l}$ ) and ECL reagents were from Amersham (Baie d'Urfé, PQ). Polyclonal rabbit antiserum raised against maltose-binding protein (anti-MBP) was purchased from New England BioLabs (Beverly, MA). Cy3-conjugated anti-rabbit IgG was purchased from Chemicon International (Temecula, CA). Unless specified otherwise, all other reagents were purchased from Sigma or Fisher. Water used for RNA procedures was double-distilled, pretreated with diethylpyrocarbonate (DEPC), and autoclaved.

\section{Experimental Animals}

Adult rainbow trout were acquired from the University of Guelph Aquaculture Research Station (Alma, $\mathrm{ON}$ ). These fish were derived from a fall-spawning stock and were raised to adulthood in outdoor tanks receiving spring water at ambient temperatures. The fish were transferred to the Hagen Aqualab at the University of Guelph, where they were maintained indoors under a simulated natural photoperiod in circular fiberglass tanks receiving re-circulated well water $\left(9 \pm 1^{\circ} \mathrm{C}\right)$. The fish were fed to satiety 3-4 times per week with a commercial trout formulation (Martin Mills, Elmira, ON). All experiments were conducted in accordance with animal use protocols approved by the Canadian Council for Animal Care.

\section{Tissue Sampling}

Ovarian tissues were collected from twelve female fish over the course of a spawning season. Three fish were sampled during the previtellogenic phase (follicle diameter (FD) $<0.1 \mathrm{~mm}$ ); three fish had earlyvitellogenic ovaries (FD $0.5-1 \mathrm{~mm}$ ); four fish had mid-vitellogenic ovaries (FD, $2-3 \mathrm{~mm}$ ), and two fish had ovaries in advanced stages of vitellogenesis (FD, 3$4 \mathrm{~mm}$ ).

The fish were anaesthetized with 2-phenoxyethanol and quickly killed by cervical transection. A subsample of ovarian tissue (follicles and stroma) was immediately frozen in liquid nitrogen, and another subsample was fixed in $4 \%$ phosphate-buffered paraformaldehyde for immunocytochemical analysis. Selected somatic tissues were also excised and frozen in liquid nitrogen; these included liver, kidney, heart, spleen, white muscle, brain, intestine, gill, and ovary. Additionally, from a 
subset of vitellogenic fish, we isolated and snap-froze selected follicles that displayed morphological features indicative of atresia (collapsed follicular wall, increased vascularization).

\section{RNA Extraction}

Total RNA was extracted from ovarian tissue homogenates using Trizol Reagent, according to supplier's instructions. Purified total RNA was resuspended in an appropriate volume of sterile, DEPC-treated water, and quantified by absorbance at $260 \mathrm{~nm}$.

\section{First-Strand Synthesis (for RT-PCR)}

Aliquots of total RNA (500 ng) from individual tissues were combined with $0.1 \mathrm{ng}$ of random hexamers primers, brought to a final volume of $12 \mu \mathrm{l}$ in DEPC-treated water, incubated for $5 \mathrm{~min}$ at $70^{\circ} \mathrm{C}$, and transferred to ice. The volume of each reaction mixture was brought to $25 \mu \mathrm{l}$ by the addition of (concentrations/activities represent final values): $5 \times \mathrm{RT}$ buffer $(50 \mathrm{mM}$ Tris- $\mathrm{HCl}$, $75 \mathrm{mM} \mathrm{KCl}, 3 \mathrm{mM} \mathrm{MgCl}_{2}$ ), DTT $(10 \mathrm{mM})$, dNTPs $(0.5 \mathrm{mM})$, RNase inhibitor (25 U), Moloney murine leukemia virus reverse transcriptase $(200 \mathrm{U})$, and DEPC-treated dd $\mathrm{H}_{2} \mathrm{O}$. Samples were incubated for $1 \mathrm{hr}$ at $37^{\circ} \mathrm{C}$, followed by $5 \mathrm{~min}$ at $90^{\circ} \mathrm{C}$ to inactive the reverse transcriptase. Reaction products were stored at $-20^{\circ} \mathrm{C}$ until PCR amplification. To confirm the absence of genomic DNA contamination, equivalent RNA samples from each tissue were treated in parallel to similar reaction conditions, but without the addition of the reverse transcriptase enzyme.

\section{PCR Amplification}

For initial amplification of the target gene (termed "oocyte protease inhibitor-1," OPI-1), we designed an oligonucleotide primer pair (RT-OPI-F1, RT-OPI-R1; Table 1) that flanked a 281 base-pair (bp) region (positions 29 to 310) of the full-length cDNA sequence of brook trout OCPI (Accession no. AF223387). For subsequent amplification of a cDNA spanning the complete open reading frame, a second antisense primer (RT-OPI-R2) was designed, downstream of the predicted stop codon.

Each PCR reaction tube contained $12.5 \mu \mathrm{l}$ of firststrand cDNA, PCR nucleotide mix $(0.2 \mathrm{mM})$, primers $(0.4 \mu \mathrm{m}$ each), PCR reaction buffer $(10 \mathrm{mM}$ Tris- $\mathrm{HCl}$, $50 \mathrm{mM} \mathrm{KCl}, 1.5 \mathrm{mM} \mathrm{MgCl}_{2}$ ), and TAq polymerase (2.5 U), diluted in sterile $\mathrm{ddH}_{2} \mathrm{O}$ to a volume of $100 \mu \mathrm{l}$. An initial denaturing step $\left(94^{\circ} \mathrm{C}, 10 \mathrm{~min}\right)$ was followed by 35 cycles in the following conditions: denaturation

TABLE 1. Oligonucleotide Primers Used in This Study

\begin{tabular}{ll}
\hline Primer name & \multicolumn{1}{c}{ Primer sequence } \\
\hline RT-OPI-F1 & 5'-GGAAGAGTGCAGAAGGAGAC-3' \\
RT-OPI-R1 & 5'-AGCAGTGCCTGGTGGAGTCT-3' \\
RT-OPI-R2 & 5'-AACATCTTCATTCCAACGCC-3' \\
RT-OPI-2-F1 & 5'-CCACCCAGAATGGTATGATACGACC-3' \\
Actin-F1 & 5'-ATGGGCCAGAAAGACAGCTA-3' \\
Actin-R1 & 5'-CCAGAGGCGTATAGGGACAA-3' \\
\hline
\end{tabular}

$\left(94^{\circ} \mathrm{C}\right.$ for $\left.1 \mathrm{~min}\right)$, annealing $\left(60^{\circ} \mathrm{C}\right.$ for $\left.1 \mathrm{~min}\right)$, and extension $\left(72^{\circ} \mathrm{C}\right.$ for $\left.1 \mathrm{~min}\right)$, in an Eppendorf Mastercycler ${ }^{\mathrm{B}}$ gradient thermocycler. After a terminal extension of $5 \mathrm{~min}$ at $72^{\circ} \mathrm{C}$, the reaction products were stored at $-20^{\circ} \mathrm{C}$.

To confirm the presence and integrity of RNA in each preparation, we performed parallel PCR reactions using oligonucleotide primers (Actin-F1, Actin-R1) for rainbow trout $\beta$-actin (Accession No. AJ438158). Reaction conditions were identical to those described above.

PCR products were fractionated in 1.5\% agarose gels, stained with ethidium bromide, and visualized under UV light. The molecular weights of amplification products were estimated by comparison to the migration distance of DNA molecular weight standards.

\section{3'-Rapid Amplification of cDNA Ends (RACE) PCR}

The above PCR reactions led to the identification of two cDNA sequences (see Results). The longer of these sequences contained an incomplete open reading frame, so we employed 3 -RACE PCR to further determine the sequence of this cDNA.

Total RNA (500 ng) and GeneRacer ${ }^{\mathrm{TM}}$ Oligo dT primers $(0.1 \mathrm{ng})$ were diluted to $12 \mu \mathrm{l}$ in sterile, DEPCtreated water. Each tube was incubated for $5 \mathrm{~min}$ at $65^{\circ} \mathrm{C}$, transferred to ice, and the volume of each reaction was brought to $20 \mu \mathrm{l}$ by the addition of $5 \times \mathrm{RT}$ Buffer (50 mM Tris- $\mathrm{HCl}, 75 \mathrm{mM} \mathrm{KCl}, 3 \mathrm{mM} \mathrm{MgCl}_{2}$ ), DTT $(10 \mathrm{mM})$, dNTPs $(0.5 \mathrm{mM})$, RNase inhibitor (25 U), SuperScript $^{\mathrm{TM}}$ II reverse-transcriptase $(200 \mathrm{U})$ and DEPC-treated $\mathrm{ddH}_{2} \mathrm{O}$. Reaction tubes were incubated for $1 \mathrm{hr}$ at $42^{\circ} \mathrm{C}$, followed by $15 \mathrm{~min}$ at $70^{\circ} \mathrm{C}$. Samples were then digested for $15 \mathrm{~min}$ at $37^{\circ} \mathrm{C}$ with RNase $\mathrm{H}$ $(2 \mathrm{U})$, and the reaction products were stored at $-20^{\circ} \mathrm{C}$. The absence of genomic DNA contamination was confirmed as described above.

3'-RACE PCR reaction mixtures contained $2 \mu \mathrm{l}$ of $3^{\prime}$-RACE cDNA, the GeneRacer ${ }^{\mathrm{TM}} 3^{\prime}$ oligonucleotide primer (supplied), a gene specific primer (RT-OPI-2-F1), and reagents as specified in the manufacturer's instructions (GeneRacer ${ }^{\mathrm{TM}}$ Kit). PCR cycle conditions were identical to those described above.

\section{Cloning and Sequencing}

Where possible, PCR products were directly sequenced by the terminal dye-labeling method on an ABI automated sequencer (Applied Biosystems, Foster City, CA). Products were first purified using the QIAquick ${ }^{\mathrm{R}}$ PCR Purification Kit (QIAGEN, Inc., Mississauga, ON), and then sequenced in both directions using the primers designed for their initial amplification. Sequences were confirmed by sequencing reaction products from at least three different fish. If direct sequencing was not possible (i.e., multiple amplification products), then the desired band was excised from the gel, purified (QIAquick ${ }^{\mathbb{R}}$ Gel Extraction Kit, QIAGEN, Inc.), inserted into the $\mathrm{pCR}^{\circledR}$ 2.1-TOPO ${ }^{\circledR}$ vector (Invitrogen) and transfected into competent TOP10 One Shot ${ }^{\circledR}$ chemically competent cells (Invitrogen). Recombinants were identified 
through blue-white color selection, grown overnight at $37^{\circ} \mathrm{C}$ in selective medium (LB broth with ampicillin, $50 \mu \mathrm{g} / \mathrm{ml}$ ), and plasmid DNA was purified using the QIAPrep ${ }^{\circledR}$ Mini Plasmid Identification Kit (QIAGEN, Inc.). Automated sequencing of individual clones was performed using M13 sequencing primers.

\section{Semi-Quantitative RT-PCR}

Semi-quantitative RT-PCR was employed to test the hypothesis that mRNA expression of OPI-1 is downregulated in atretic follicles as compared to healthy vitellogenic follicles. First-strand synthesis, in $100 \mu \mathrm{l}$ volumes, was performed as described above, using total RNA from either healthy mid-vitellogenic follicles, or morphologically atretic follicles. PCR amplification of OPI-1 was performed with the primer pair RT-OPI-F1 and RT-OPI-R2, and rainbow trout $\beta$-actin was used as the reference standard. Reaction tubes containing equal volumes of first-strand cDNA from each sample were amplified for 12-36 PCR cycles (in 2-3 cycle increments), electrophoretically fractionated and stained with ethidium bromide. PCR amplification products were quantified densitometrically using digital imaging software (IS-1000; Alpha Innotech Corp., San Leandro, CA). The relative expression levels of OPI-1 in vitellogenic and atretic follicles were determined by comparing the ratio of the densities of OPI- 1 and $\beta$-actin amplification products after an appropriate number of PCR cycles, ensuring amplification of each target sequence was terminated during the exponential phase of amplification; this was quantitatively determined, and shown to be consistent between samples. Comparisons were made after 18 cycles for $\beta$-actin, and 27 cycles for OPI-1, and each reaction was repeated three times with similar results.

\section{Western Immunoblotting}

We used Western immunoblotting to visualize proteins encoded by the PCR-amplified genes. Vitellogenic ovarian follicles were punctured and samples of whole yolk were diluted 1:100 in SDS-PAGE sample buffer (1\% SDS, 0.1 M Tris-HCl, 10\% glycerol, $0.02 \%$ bromophenol blue, $2 \%$ 2-mercaptoethanol), heated for $10 \mathrm{~min}$ at $65^{\circ} \mathrm{C}$, electrophoretically separated on $14 \%$ polyacrylamide gels, and transferred for $30 \mathrm{~min}$ to Immobilon-P PVDF membranes (Millipore, Milwaukee, WI). After transfer, the membranes were incubated for $2 \mathrm{hr}$ at room temperature in blotting buffer (phosphate-buffered saline, $0.1 \%$ Tween ${ }^{\circledR} 20,3 \%$ BSA, pH 7.4), and then blotted overnight at $4{ }^{\circ} \mathrm{C}$ with rabbit antiserum $(1: 1,000$ in blotting buffer) raised against a recombinantly expressed maltose-binding protein:brook trout OCPI fusion protein (anti-OCPI) (Bobe and Goetz, 2001), generously provided by Dr. Frederick W. Goetz (Marine Biological Laboratories, Wood's Hole, MA). Membranes were then blotted for $2 \mathrm{hr}$ at room temperature with secondary antibody (HRP-conjugated goat anti-rabbit IgG, 1:5,000 in blotting buffer) and immunoreactive proteins were visualized using the ECL detection system. Negative controls were performed by immuno- blotting with rabbit antiserum $(1: 1,000)$ raised against maltose-binding protein (anti-MBP).

\section{Immunocytochemistry}

Immunocytochemistry was used to determine the spatial expression patterns of target proteins in situ. Paraformaldehyde-fixed ovarian tissues were dehydrated in a graded ethanol series, embedded in paraffin, and sectioned at $4 \mu \mathrm{m}$. After deparaffinizing (1 hr at $60^{\circ} \mathrm{C}$ ), the sections were cleared in xylenes, rehydrated in a graded ethanol series, and washed three times in phosphate-buffered saline containing $0.1 \%$ Tween 20 (PBST, pH 7.4). Sections were then preincubated for $2 \mathrm{hr}$ at room temperature in blocking buffer $(0.2 \% \mathrm{BSA}, 2 \%$ goat serum, $1 \%$ DMSO, in PBST), followed by overnight $\left(4{ }^{\circ} \mathrm{C}\right.$ ) incubation in primary antiserum (anti-OCPI, diluted 1:1,000 in blocking buffer). Two control groups were included: (1) a subset of slides were incubated with control antibody (anti-MBP, 1:1,000); and (2) a subset of slides were incubated in blocking buffer without primary antibody. All slides were then washed $3 \times$ $10 \mathrm{~min}$ in PBST, and incubated for $2 \mathrm{hr}$ at room temperature in secondary antibody (Cy3-conjugated antirabbit IgG; 1:500 in blocking buffer). The sections were then washed $(3 \times 10 \mathrm{~min}$ in PBST $)$ and examined under fluorescence and differential interference contrast microscopy. Digital images were captured using Scion Image software (National Institutes of Health, Bethesda, MA).

\section{Data Analyses}

Cloned sequences were submitted to public database searches (BLAST) to identify similar published sequences. Multiple sequence alignments were generated using ClustalW, and protein sequence analyses were performed with the Sequence Manipulation Suite (http://www.bioinformatics.org/SMS/), the Simple Modular Architecture Research Tool (Letunic et al., 2002) and Pfam (http://pfam.wustl.edu/). The relative mRNA expression levels of OPI-1 mRNA in healthy vitellogenic and atretic follicles were statistically compared using an unpaired Student's $t$-test. Differences were judged to be significant if $P<0.05$.

\section{RESULTS \\ Sequence Identification}

RT-PCR of total RNA from rainbow trout midvitellogenic ovaries using the primer pair RT-OPI-F1 and RT-OPI-R1 yielded a single 279-bp cDNA (Fig. 1, lane 2). This sequence showed high (94\%) identity to brook trout OCPI, confirming that we had successfully amplified the target sequence. This sequence contained an ATG site at position 28, flanked by the Kozak initiation sequence (ACCATGG) (Kozak, 1986), but did not contain an in-frame stop codon. RT-PCR of total RNA from rainbow trout mid-vitellogenic ovaries using the primer pair RT-OPI-F1 and RT-OPI-R2 yielded a single 330-bp cDNA (Fig. 1, lane 3). This sequence was identical to the initial transcript in corresponding 


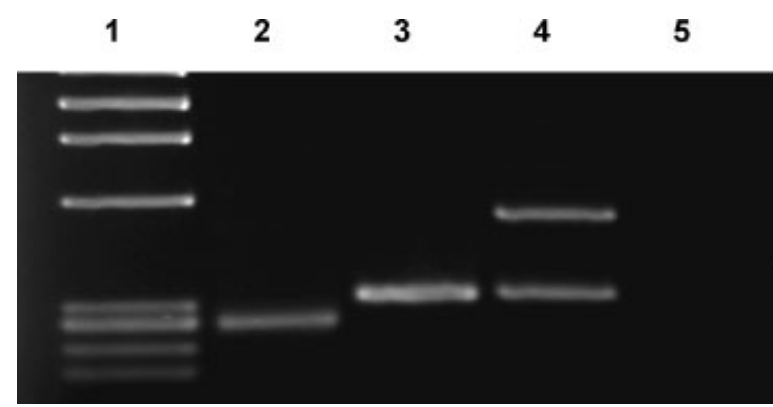

Fig. 1. Reverse transcription-polymerase chain reaction (RT-PCR) products amplified from total RNA of rainbow trout vitellogenic oocytes. Lane 1, molecular weight standard; lane 2, rainbow trout OPI-1 partial cDNA (279 bp; amplified using RT-OPI-F1 and RT-OPIR1); lane 3, rainbow trout OPI-1 cDNA (330 bp; amplified using RTOPI-F1 and RT-OPI-R2); lane 4, OPI-2 partial cDNA (upper band, 548 bp; amplified using RT-OPI-F1 and RT-OPI-R2); lane 5, negative control (no first-strand cDNA).

regions, and contained an in-frame stop codon (TAG) at position 289.

RT-PCR of total RNA from early-vitellogenic ovarian tissues, using either primer pair, frequently yielded two amplification products. Using the second primer pair (RT-OPI-F1 and RT-OPI-R2), these amplicons were approximately 330 and 550 bp (Fig. 1, lane 4). The shorter amplicon was identical in sequence to the $330 \mathrm{bp}$ cDNA previously identified; the longer sequence was revealed to be a partial cDNA that resembled a tandem duplication of the shorter cDNA, but which did not contain an in-frame stop codon. 3'-RACE PCR, using the GeneRacer $3^{\prime}$ primer and a gene-specific primer (RT-OPI-2-F1), using total RNA purified from midand late-vitellogenic ovarian tissues, generated a $420 \mathrm{bp}$ cDNA, which was subsequently cloned and sequenced. The assembled sequence (645 bp) contained an inframe stop codon at position 547, and a polyadenylation signal.

\section{Sequence Analysis and Tissue Localization}

The open reading frame (nucleotides 28-288) of the shorter $(330 \mathrm{bp}) \mathrm{cDNA}$ is predicted to encode an 87 amino acid peptide (Fig. 2), with a calculated molecular mass of $9.1 \mathrm{kDa}$. The deduced amino acid sequence has high identity to chum salmon ECI-3, and the deduced



Fig. 2. Rainbow trout OPI-1 (Accession No. AY094359) cDNA sequence, with translation to OPI-1 protein. Start and stop codons are indicated with bold-faced text; putative thyroglobulin type-1 domain is indicated with shaded text.

amino acid sequences of brook trout OCPI and Atlantic salmon cys protease inhibitor (Table 2). The rainbow trout sequence contains a single TY motif, spanning residues 27-87 (Fig. 3a) (Marchler-Bauer et al., 2002). Based on the presence of this single TY motif, we have termed this cDNA sequence "oocyte protease inhibitor1" (OPI-1, GenBank Accession No. AY094359).

Using the initial primer pair (RT-OPI-F1, RT-OPIR1), we amplified OPI-1 from early-, mid-, and latevitellogenic ovarian tissues, but were unable to amplify OPI-1 from liver, kidney, spleen, brain, muscle, gill, or heart (Fig. 4, upper panel). We were also unable to amplify OPI-1 using total RNA purified from previtellogenic ovarian tissues ( $\mathrm{n}=3$ fishes; Fig. 4, upper panel). $\beta$-actin was abundantly amplified from each sample, confirming the presence and integrity of first-strand cDNA in each tissue (Fig. 4, bottom panel).

The open reading frame (nucleotides 28-546) of the longer $(645 \mathrm{bp}) \mathrm{cDNA}$ is predicted to encode a 173 amino acid peptide (Fig. 5), containing two TY domains spanning residues $27-88$ and 101-162, respectively (Fig. 3a). These domains are similar but not identical, and neither domain is identical to the TY domain in OPI-1 (Fig. 3b). Based on the presence of these two TY domains, we have named this sequence "oocyte protease inhibitor-2" (OPI-2; Accession No. AF529226).

TABLE 2. Comparison of Rainbow Trout OPI-1 (AY094359) Amino Acid Sequence With Putative Homologs Identified in Atlantic Salmon, Brook Trout, and Chum Salmon

\begin{tabular}{lccc}
\hline Gene name & Accession no. & Identity (\%) & Similarity (\%) \\
\hline $\begin{array}{l}\text { Atlantic salmon cys protease } \\
\text { inhibitor }\end{array}$ & BE518516 & $97^{\mathrm{a}}$ & $99^{\mathrm{a}}$ \\
$\begin{array}{l}\text { Atlantic salmon cys protease } \\
\text { inhibitor }\end{array}$ & BE518515 & $72^{\mathrm{a}}$ & $83^{\mathrm{a}}$ \\
$\begin{array}{l}\text { Brook trout oocyte cysteine } \\
\text { protease inhibitor (OCPI) }\end{array}$ & AF223387 & $88^{\mathrm{a}}$ & $92^{\mathrm{a}}$ \\
$\begin{array}{c}\text { Chum salmon egg cysteine } \\
\text { protease inhibitor (ECI-1) }\end{array}$ & $2207269 \mathrm{~A}$ & 63 & 84 \\
\hline
\end{tabular}

${ }^{\mathrm{a} A m i n o}$ acid sequences predicted from nucleotide sequence. 

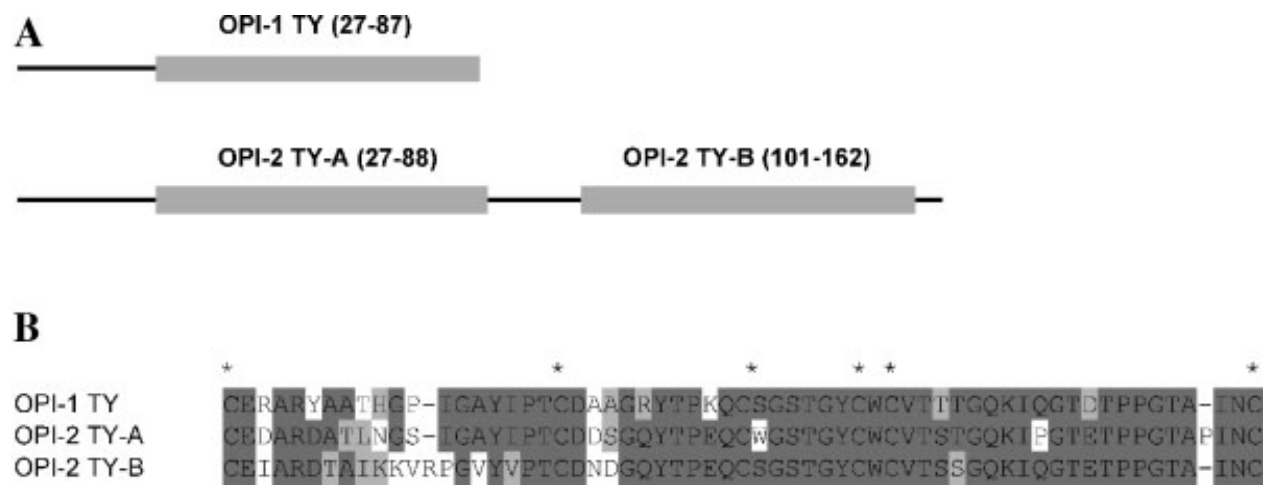

Fig. 3. A: Schematic diagram indicating the cysteine-rich thyroglobulin type-1 (TY) motifs in rainbow trout OPI-1 and OPI-2. Corresponding amino acid residues are indicated in parentheses. B: ClustalW alignments of thyroglobulin type-1 domains in rainbow trout OPI-1 and OPI-2. Asterisks denote the location of domain-specific cysteine residues.

The initial RT-PCR reactions confirmed that OPI-2 mRNA is expressed in the early vitellogenic ovary, and the gene-specific RACE-PCR results revealed expression of this transcript in mid- and late-vitellogenic ovaries. Furthermore, since our initial primer pairs (e.g., RT-OPI-F1, RT-OPI-R2) were shown to effectively amplify OPI-2 if present, our results demonstrate that OPI-2 mRNA is not detectably expressed in the previtellogenic ovary, or in any of the somatic tissues examined (Fig. 4).

\section{Immunoblotting}

Western immunoblots of ooplasm from vitellogenic oocytes using antiserum raised against brook trout OCPI revealed two immunopositive bands, of approximately 10 and $22 \mathrm{kDA}$ (Fig. 6). These bands are similar in size to the predicted molecular weights of rainbow trout OPI-1 $(9.1 \mathrm{kDa})$ and OPI-2 $(18.3 \mathrm{kDa})$. Immunopositive bands were not observed on blots using antiMBP as the primary antibody (Fig. 6).

\section{Immunocytochemistry}

Immunocytochemical analyses of rainbow trout ovarian tissue sections using antiserum raised against brook trout OCPI revealed intense immunostaining in vesicles located in the cortical ooplasm of vitellogenic oocytes



Fig. 4. Tissue distribution of rainbow trout OPI-1 (upper panel) and $\beta$-actin cDNA (lower panel) as determined by reverse-transcriptase polymerase chain reaction (RT-PCR). Each lane represents cDNA amplified from $250 \mathrm{ng}$ of total RNA from (2) mid-vitellogenic oocytes, (3) brain, (4) kidney, (5) heart, (6) spleen, (7) white muscle, (8) liver, (9) gill, (10) previtellogenic ovary, and (11) follicular epithelium. Lane 1 (upper panel) contains a DNA molecular weight standard and lane 12 (both panels) contains negative controls (no cDNA template).
(Fig. 7a,b). These vesicles were distinct from the yolk vesicles that coalesce to form the yolk platelets. Since our Western immunoblot results suggest that this polyclonal antiserum crossreacts with both OPI-1 and OPI-2, it is likely that the positive immunocytochemical staining in these sections represents both proteins. Epitope-specific antibodies are not currently available for these proteins, preventing us from independently determining the subcellular localization of each protein.
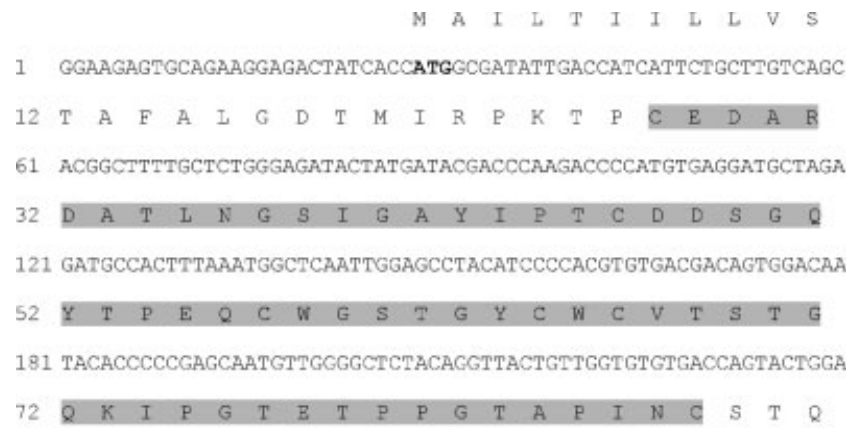

241 CAGAAGATCCCGGGTACTGAGACTCCACCAGGCACTGCTCCAATCAACTGTTCCACCCAG

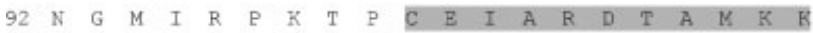
301 AATGGTATGATACGACCCAAGACCCCCTGTGAGATTGCTAGAGATACTGCGATGAMNAAA

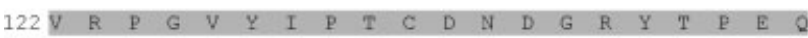
361 GTCAGGCCTGGAGTCTACATCCCCACGTGTGACAACGATGGACGATACACCCCTGAGCAA

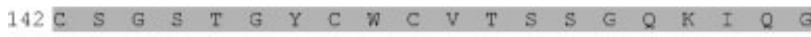
421 TGTTCAGGCTCTACAGGTTACTGTTGGTGTGTGACCAGTTCTGGACAGAAGATCCAGGGT


481 ACTGAGACTCCACCAGGCACTGCTATCAACT GTTCCACCAAGATGAAAGGCGTTGGAAGT $182 \mathrm{~K} \mathrm{M}$

541 AAGATGTAGAaGACGGaCAGTTGTCTTTACTGTACTACAGGAAGGGATTCACATCCCATA

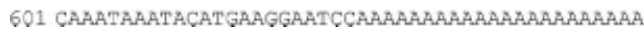

Fig. 5. Rainbow trout OPI-2 (Accession no. AF529226) cDNA sequence, with translation to OPI-2 protein. Start and stop codons are indicated with bold-faced text; putative thyroglobulin type-1 domains are indicated with shaded text. 

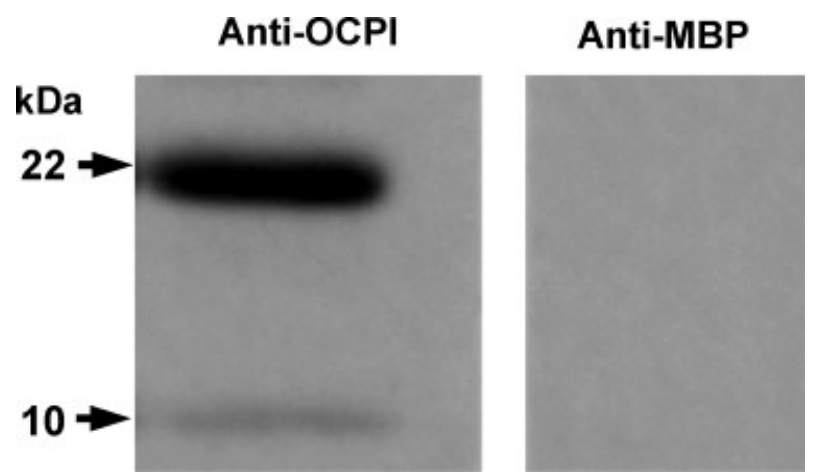

Fig. 6. Western immunoblots of rainbow trout yolk/ooplasm using (A) antiserum raised against brook trout OCPI (anti-OCPI), or (B) antiserum raised against maltose-binding protein (anti-MBP). No positive immunostaining was detected with anti-MBP (control antibody)

We did not detect any positive immunostaining in previtellogenic oocytes, nor did we detect any positive immunostaining in somatic ovarian tissues (Fig. 7a,b). Furthermore, no positive immunostaining was detected in tissue sections incubated with secondary antibody alone (results not shown). In some tissue sections, we detected faint crossreactivity of the control antiserum (anti-MBP) with ovarian vascular tissues (Fig. 7c,d); we did not, however, detect any positive immunostaining that corresponded to the positive immunostaining detected with OCPI antiserum.
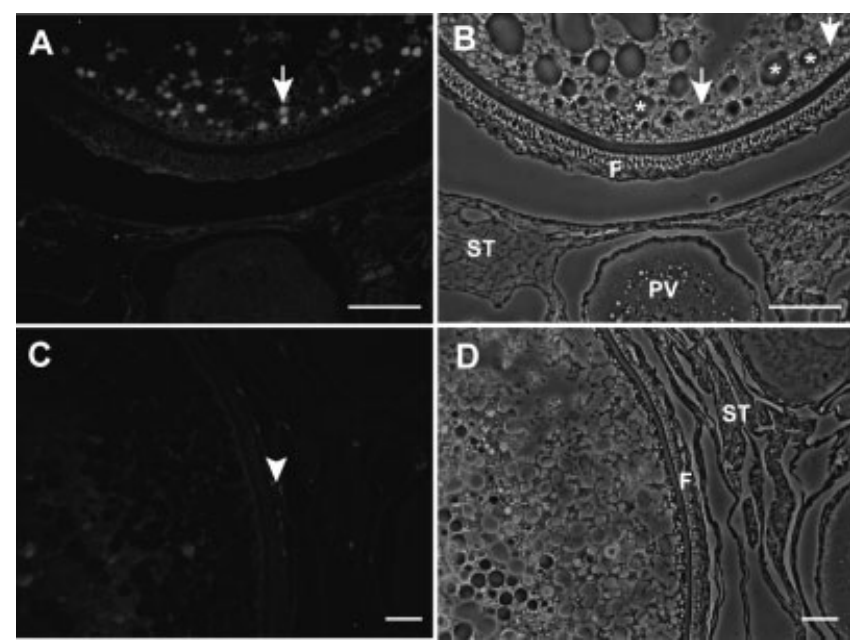

Fig. 7. Fluorescence $(\mathbf{A}, \mathbf{C})$ and differential interference contrast (B, D) microscopy images of rainbow trout ovarian sections immunocytochemically stained with antiserum raised against (A) brook trout OCPI (anti-OCPI), or (C) maltose-binding protein (anti-MBP). Positive immunostaining for rainbow trout OPI(s) (A) is detected in vesicles in the cortical ooplasm (arrows), but is not detected in yolk spheres (asterisks), previtellogenic oocytes (PV), stromal tissue (ST), or the somatic follicular epithelium (F). Weak crossreactivity of anti-MBP (control) antiserum was detected in the ovarian vasculature (arrowhead), but no positive immunostaining was detected in the ooplasm. Scale bars indicate $100 \mu \mathrm{m}$.

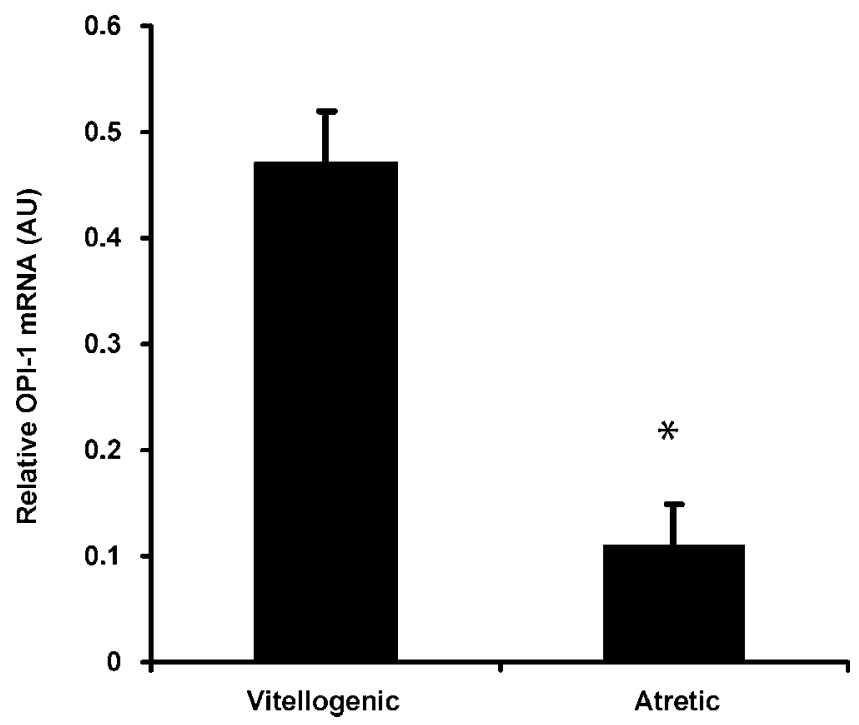

Fig. 8. Relative mRNA expression of rainbow trout OPI-1 in vitellogenic versus atretic oocytes. Values represent the mean $\pm \mathrm{SEM}$ of the ratios of OPI- 1 expression to $\beta$-actin expression as determined by semi-quantitative RT-PCR. Asterisk indicates a statistically significant difference in relative OPI-1 expression between vitellogenic and atretic oocytes $(P<0.05)$.

\section{OPI-1 mRNA Expression in Vitellogenic Versus Atretic Follicles}

The amplification profile of $\beta$-actin with increasing PCR cycle numbers (12-27) was consistent between healthy and atretic oocytes (data not shown), confirming that $\beta$-actin was an appropriate internal standard by which to determine the relative expression of OPI-1. Using this approach, we determined that OPI-1 mRNA expression was significantly lower in atretic oocytes as compared to healthy oocytes $(t$-test, $P<0.001, \mathrm{n}=3$ (Fig. 8).

\section{DISCUSSION}

In this study, we report the identification and characterization of two oocyte protease inhibitor cDNAs in rainbow trout ovary. One of these cDNAs (OPI-1) is predicted to encode the rainbow trout homolog of an OPI recently identified in related teleosts (Yamashita and Konagaya, 1996; Bobe and Goetz, 2001; Davey et al., 2001). The second cDNA (OPI-2) is a novel gene that resembles a tandem duplication of OPI-1, but which has not previously been reported. Herein, we provide the first data describing the temporal and spatial expression profiles of both genes throughout the vitellogenic growth phase of oocyte development. As hypothesized, these genes were shown to be expressed exclusively during vitellogenesis, in subcellular regions directly associated with the uptake and processing of yolk lipoproteins (Sire et al., 1994).

Both cDNAs contain at least one TY domain, encoding a functional motif suggested to be the hallmark of a novel class of protease inhibitors called "thyroglobulin type-1 domain inhibitors" (Lenarcic and Turk, 1999). 
This motif is found, often in repeats, among a variety of different proteins, including thyroglobulin (11 TY repeats), nidogen (2 TY repeats), sea anemone equistatin (3 TY repeats), bullfrog saxiphilin (2 TY repeats), and all insulin-like growth factor binding proteins (1 TY repeat each). While these proteins do not all function exclusively as protease inhibitors, the presence of this domain has been shown to confer resistance against endogenous proteases, particularly cathepsins and cathepsin-like proteases (Yamashita and Konagaya, 1991, 1996; Fowlkes et al., 1997; Lenarcic et al., 1997; Lenarcic and Turk, 1999).

Multiple studies have identified cathepsin proteases as key mediators of yolk processing during vitellogenic oocyte development in a wide variety of species (Fagotto, 1990; Nakamura et al., 1996; De Stasio et al., 1999; Gerhartz et al., 1999; Carnevali et al., 1999b). What remains enigmatic are the mechanisms by which multiple cathepsin proteases are differentially controlled during key developmental events involving proteolysis. These events include the partial breakdown of complex yolk lipoprotein precursors (e.g., vitellogenin) into yolk storage proteins, the proteolytic rupture of follicles during ovulation, and the subsequent hydrolysis of yolk storage proteins that is required to generate metabolic substrate for embryonic development. The identification of rainbow trout OPI-1 and OPI-2, collectively possessing three distinct TY domains, suggests a mechanism to explain how the activity of multiple related proteases may be differentially regulated during vitellogenic oocyte development. This proposed mechanism is supported by data from Lenarcic and Turk (1999), who demonstrated that the three distinct TY domains in sea anemone equistatin exhibit differential binding affinities and inhibitory activities against specific cathepsins and cathepsin-like proteases, despite high sequence similarities between the domains.

The data from this study provide three lines of evidence in support of our hypothesis that OPI-1 and OPI-2 are TY domain inhibitors that function to regulate yolk protease activity during vitellogenic oocyte development. Firstly, we have demonstrated the onset of ovary-specific mRNA expression of both genes at the onset of vitellogenin uptake in rainbow trout. Secondly, we have shown that the proteins encoded by these transcripts are specifically localized to defined vesicles in the cortical ooplasm of vitellogenic oocytes. This region of the oocyte has previously been confirmed as the location of vitellogenin processing in avian (Ito et al., 2003), amphibian (Wall and Patel, 1987), and piscine species (Sire et al., 1994). Thirdly, we have demonstrated a significant decline in mRNA expression of OPI-1 in atretic oocytes, relative to healthy vitellogenic oocytes. These data are consistent with our hypothesis, and are concordant with data from earlier studies demonstrating increased cathepsin-like protease activity in degenerating oocytes (Kestemont et al., 1999; Wood and Van Der Kraak, 2003). We suggest that decreased expression of TY domain protease inhibitors at the onset of atresia would facilitate the proteolytic degradation and resorption of yolk proteins, events which are strongly associated with atresia in oviparous vertebrates (Saidapur, 1978).

The expression pattern of rainbow trout OPI-1 differs somewhat from the reported expression pattern of the brook trout homolog (OCPI). Bobe and Goetz (2001) detected brook trout OCPI mRNA and protein in what were described as previtellogenic follicles, whereas we were unable to detect OPI-1 expression in the previtellogenic ovary, using both immunocytochemistry and the more sensitive RT-PCR technique. One plausible explanation for these divergent observations relates to the specific stages of ovarian development of fish used in each study. In sexually mature female salmonids, there are typically two cohorts of follicles present in the ovary: one cohort consists of actively growing follicles recruited for growth and maturation in the current season, while the second cohort consists of quiescent (non-growing) primary follicles. The latter population remains in an arrested state until recruitment to the vitellogenic growth phase, following ovulation of the mature cohort. Bobe and Goetz (2001) restricted their examination of OCPI expression to periovulatory and postovulatory ovaries. Thus the "previtellogenic" follicles examined in that study had likely been recruited to the next season's cohort of growing follicles, in which case vitellogenesis would likely have commenced. By contrast, the previtellogenic follicles examined in our RT-PCR analyses were from pre-pubertal fish, allowing us to confirm that vitellogenesis had not yet begun.

The fortuitous identification of OPI-2 using primers designed to amplify OPI-1 was the result of sequence similarities between the two cDNAs. Sequence comparisons of OPI-1 and OPI-2 cDNAs, however, indicate that these transcripts are not splice variants of a single gene, but rather represent transcription products derived from separate genes. We have hypothesized that these genes are paralogous, though it is unclear at this time which was the original duplicated gene. Sequence comparisons revealed greater similarity between the TY domain of OPI-1 and the first TY domain of OPI-2 (Fig. 3b), but these data alone cannot resolve the origin of these paralogs. It also remains unknown if this putative duplication occurred within the species lineage, or whether other species possess an ortholog to OPI-2; to date, none has been identified.

Our RT-PCR results demonstrating mRNA expression of OPI-1, but not OPI-2, at some stages of development were likely the result of preferential PCR amplification of the shorter (OPI-1) transcript, as our RACE-PCR results confirmed that OPI- 2 mRNA is expressed throughout the vitellogenic phase of oocyte growth. In fact, the Western immunoblot results suggest that OPI-2 protein may be more abundantly expressed than OPI-1 protein in vitellogenic oocytes. Alternatively, the stronger immunogenic signal for OPI-2 may simply represent greater crossreactivity of the antiserum with the repetitive domains of OPI-2. 
In summary, we have identified two putative TY domain inhibitors in rainbow trout ovary whose expression patterns are strongly associated with the vitellogenic phase of oocyte growth. This is the first study to demonstrate expression of multiple TY domain inhibitors in the developing teleost ovary, and the first to demonstrate their expression at multiple stages of ovarian development. Messenger RNA expression of both genes was detected in ovarian tissues throughout the vitellogenic phase, but was significantly reduced in degenerating (atretic) oocytes. The proteins encoded by these transcripts are localized to the cortical ooplasm of vitellogenic oocytes, in direct association with sites of vitellogenin processing. The identification of these genes provides new evidence for the involvement of multiple factors in regulating yolk processing during vitellogenesis in teleosts.

\section{ACKNOWLEDGMENTS}

The thoughtful contributions of Dr. Karim Gharbi and Dr. Nicholas Bernier (University of Guelph) are very much appreciated. The authors are grateful to Michael Burke and staff of the Alma Aquaculture Research Station for providing experimental fish. This study was funded by an operating grant to G.V.D.K. from the Natural Sciences and Engineering Research Council (NSERC) of Canada. A.W.W. received financial support from NSERC and the Ontario Graduate Scholarship program. The authors thank Dr. Cunming Duan (University of Michigan) and Dr. Erica Crespi (University of Michigan) for helpful contributions to this manuscript.

\section{REFERENCES}

Bobe J, Goetz FW. 2001. Cysteine protease inhibitor is specifically expressed in pre- and early-vitellogenic oocytes from the brook trout periovulatory ovary. Mol Reprod Dev 60:312-318.

Carnevali O, Carletta R, Cambi A, Vita A, Bromage N. 1999a. Yolk formation and degradation during oocyte maturation in seabream Sparus aurata: Involvement of two lysosomal proteinases. Biol Reprod 60:140-146.

Carnevali O, Centonze F, Brooks S, Marota I, Sumpter JP. 1999b. Molecular cloning and expression of ovarian cathepsin D in Seabream, Sparus aurata. Biol Reprod 61:785-791.

Coffman MA, Goetz FW. 1998. Trout ovulatory proteins are partially responsible for the anti-proteolytic activity found in trout coelomic fluid. Biol Reprod 59:497-502.

Curry TEJ, Wheeler SE. 2002. Cellular localization of tissue inhibitors of metalloproteinases in the rat ovary throughout pseudopregnancy. Biol Reprod 67:1943-1951.

Davey GC, Caplice NC, Martin SA, Powell R. 2001. A survey of genes in the Atlantic salmon (Salmo salar) as identified by expressed sequence tags. Gene 263:121-130.

De Stasio R, Borrelli L, Kille P, Parisi E, Filosa S. 1999. Isolation, characterization and molecular cloning of cathepsin $\mathrm{D}$ from lizard ovary: Changes in enzyme activity and mRNA expression throughout ovarian cycle. Mol Reprod Dev 52:126-134.

Fagotto F. 1990. Yolk degradation in tick eggs: I. Occurrence of a cathepsin L-like acid proteinase in yolk spheres. Arch Insect Biochem Physiol 14:217-235.

Fowlkes JL, Thrailkill KM, George-Nascimento C, Rosenberg CK, Serra DM. 1997. Heparin-binding, highly basic regions within the thyroglobulin type-1 repeat of insulin-like growth factor (IGF)binding proteins (IGFBPs)-3, -5 , and -6 inhibit IGFBP-4 degradation. Endocrinology 138:2280-2285.
Gerhartz B, Kolb HJ, Wittmann J. 1999. Proteolytic activity in the yolk sac membrane of quail eggs. Comp Biochem Physiol A Mol Integr Physiol 123:1-8.

Hiramatsu N, Ichikawa N, Fukada H, Fujita T, Sullivan CV, Hara A. 2002. Identification and characterization of proteases involved in specific proteolysis of vitellogenin and yolk proteins in salmonids. J Exp Zool 292:11-25.

Ito Y, Kihara M, Nakamura E, Yonezawa S, Yoshizaki N. 2003 Vitellogenin transport and yolk formation in the quail ovary. Zool Sci 20:717-726.

Kestemont P, Cooremans J, Abi-Ayad A, Mélard C. 1999. Cathepsin L in eggs and larvae of perch, Perca fluviatilis: Variations with developmental stage and spawning period. Fish Physiol Biochem 21:59-64

Kozak M. 1986. Point mutations define a sequence flanking the AUG initiator codon that modulates translation by eukaryotic ribosomes. Cell 44:283-292.

Krieger J, Fleig R. 1999. Yolk mobilization in perch, Perca fluviatilis L., embryos. Fish Physiol Biochem 21:157-165.

Kwon JY, Prat F, Randall C, Tyler CR. 2001. Molecular characterization of putative yolk processing enzymes and their expression during oogenesis and embryogenesis in rainbow trout (Oncorhynchus mykiss). Biol Reprod 65:1701-1709.

Lenarcic B, Turk V. 1999. Thyroglobulin type-1 domains in equistatin inhibit both papain-like cysteine proteinases and cathepsin D. J Biol Chem 274:563-566.

Lenarcic B, Ritonja A, Strukelj B, Turk B, Turk V. 1997. Equistatin, a new inhibitor of cysteine proteinases from Actinia equina, is structurally related to thyroglobulin type-1 domain. J Biol Chem 272:13899-13903.

Letunic I, Goodstadt L, Dickens NJ, Doerks T, Schultz J, Mott R, Ciccarelli F, Copley RR, Ponting CP, Bork P. 2002. Recent improvements to the SMART domain-based sequence annotation resource. Nucleic Acids Res 30:242-244.

Li F, An H, Seymour TA, Bradford CS, Morrissey MT, Bailey GS, Helmrich A, Barnes DW. 1998. Molecular cloning, sequence analysis and expression distribution of rainbow trout (Oncorhynchus mykiss) cystatin C. Comp Biochem Physiol B Biochem Mol Biol 121:135143.

Marchler-Bauer A, Panchenko AR, Shoemaker BA, Thiessen PA, Geer LY, Bryant SH. 2002. CDD: A database of conserved domain alignments with links to domain three-dimensional structure. Nucleic Acids Res 30:281-283.

Molina F, Bouanani M, Pau B, Granier C. 1996. Characterization of the type-1 repeat from thyroglobulin, a cysteine-rich module found in proteins from different families. Eur J Biochem 240:125133.

Nakamura K, Yonezawa S, Yoshizaki N. 1996. Vitellogenesis-related ovary cathepsin D from Xenopus laevis: Purification and properties in comparison with liver cathepsin D. Comp Biochem Physiol B Biochem Mol Biol 113:835-840.

Ribolla PEM, Bijovsky AT, de Bianchi AG. 2001. Procathepsin and acid phosphatase are stored in Musca domestica yolk spheres. J Insect Physiol 47:225-232.

Saidapur SK. 1978. Follicular atresia in the ovaries of nonmammalian vertebrates. Int Rev Cytol 54:225-244.

Sire M-F, Babin PJ, Vernier J-M. 1994. Involvement of the lysosomal system in yolk protein deposit and degradation during vitellogenesis and embryonic development in trout. J Exp Zool 269:69-83.

Tata JR, Smith DF. 1979. Vitellogenesis: A versatile model for hormonal regulation of gene expression. Rec Prog Horm Res 35:47-95.

Tyler CR, Sumpter JP. 1996. Oocyte growth and development in teleosts. Rev Fish Biol Fish 6:287-318.

Tyler CR, Sumpter JP, Bromage NR. 1990. An in vitro culture system for studying vitellogenin uptake into ovarian follicles of the rainbow trout, Salmo gairdneri. J Exp Zool 255:216-231.

Tyler CR, Sumpter JP, Kawauchi H, Swanson P. 1991. Involvement of gonadotropin in the uptake of vitellogenin into vitellogenic oocytes of the rainbow trout, Oncorhynchus mykiss. Gen Comp Endocrinol 84:291-299.

Valle D. 1993. Vitellogenesis in insects and other groups-A review. Mem Inst Oswaldo Cruz 88:1-26. 
A.W. WOOD ET AL.

Wall DA, Patel S. 1987. Multivesicular bodies play a key role in vitellogenin endocytosis by Xenopus oocytes. Dev Biol 119:275-289.

Wallace RA, Selman K. 1985. Major protein changes during vitellogenesis and maturation of Fundulus oocytes. Dev Biol 110:492-498.

Wood AW, Van Der Kraak G. 2003. Yolk proteolysis in rainbow trout oocytes after serum-free culture: Evidence for a novel biochemical mechanism of atresia in oviparous vertebrates. Mol Reprod Dev 65:219-227.

Yamamoto Y, Zhao X, Suzuki AC, Takahashi SY. 1994. Cysteine proteinase from the eggs of the silkmoth, Bombyx mori: Site of synthesis and a suggested role in yolk protein degradation. J Insect Physiol 40:447-454.

Yamashita M, Konagaya S. 1991. Cysteine protease inhibitor in egg of chum salmon. J Biochem (Tokyo) 110:762-766.

Yamashita M, Konagaya S. 1996. A novel cysteine protease inhibitor of the egg of chum salmon, containing a cysteine-rich thyroglobulin-like motif. J Biol Chem 271:1282-1284.

Yoshizaki N, Yonezawa S. 1996. Salt concentration-dependency of vitellogenin processing by cathepsin D in Xenopus laevis. Dev Growth Diff 38:549-556. 